\title{
BMJ open Chronic health problems and health-related quality of life in Chinese children and adolescents: a population-based study in Hong Kong
}

\author{
S L Lee, ${ }^{1}$ Y F Cheung, ${ }^{1}$ H S W Wong, ${ }^{1}$ T H Leung, ${ }^{2}$ T H Lam, ${ }^{3}$ Y L Lau ${ }^{1}$
}

To cite: Lee SL, Cheung YF, Wong HSW, et al. Chronic health problems and health-related quality of life in Chinese children and adolescents:

a population-based study in Hong Kong. BMJ Open 2013;3:e001183.

doi:10.1136/bmjopen-2012001183

- Prepublication history and additional material for this paper are available online. To view these files please visit the journal online (http://dx.doi.org/10.1136/ bmjopen-2012-001183).

Received 13 July 2012 Revised 23 November 2012 Accepted 26 November 2012

This final article is available for use under the terms of the Creative Commons Attribution Non-Commercial 2.0 Licence; see http://bmjopen.bmj.com

${ }^{1}$ Department of Paediatrics and Adolescent Medicine, The University of Hong Kong, Hong Kong, Hong Kong ${ }^{2}$ Department of Health, The Government of the Hong Kong, SAR, Hong Kong, Hong Kong

${ }^{3}$ School of Public Health, The University of Hong Kong, Hong Kong, Hong Kong

Correspondence to

YL Lau;

lauylung@hku.hk

\section{ABSTRACT}

Objective: We examined the association of different chronic physical and mental conditions, individually or comorbidly on health-related quality of life (QoL) in Chinese children aged $\leq 14$ years in Hong Kong.

Design: Population-based cross-sectional survey. Participants: Approximately 7500 Chinese children aged $<14$ years in Hong Kong.

Interventions: Nil.

\section{Primary and secondary outcome measures:}

Various health concepts of validated Chinese version of Child Health Questionnaire (CHQ), a health-related QoL questionnaire in children.

Result: There was significant association of physical and mental health conditions, either individually or comorbidly, on the various concepts of $\mathrm{CHQ}$. Children with mental health problems were apparently more affected than those with physical health problems. Chronic renal disease and congenital malformation were the physical health conditions associated with the lowest scores in $\mathrm{CHQ}$ concepts in children aged $5-10$ years and aged 10-14 years, respectively. Behavioural problem was the mental health condition associated with the lowest score in $\mathrm{CHQ}$ concepts in both age groups.

Conclusions: Our study shows important information concerning the prevalence of different health conditions and its association, either individually or comorbidly on the $Q o L$ in a representative sample of Chinese children in HK.

\section{INTRODUCTION}

The WHO (1948) defines health as 'a state of complete physical, mental and social wellbeing and not merely the absence of disease or infirmity'. There has been an increasing use of health-related quality of life (HRQoL) assessment tools to assess the full impact of physical illness in both children and adults to guide the attainment of possible highest standard of health at individual, societal and population level. Data from representative population sample for comparison of the impact of various conditions and for

\section{ARTICLE SUMMARY}

Article focus

- To explore any association between chronic physical and mental health conditions and quality of life (QoL) in Chinese children aged $\leq 14$ years.

- To compare the attributing effect of isolated physical health condition versus isolated mental health condition on various concepts of Child Health Questionnaire $(\mathrm{CHQ})$, a validated $\mathrm{QoL}$ questionnaire commonly employed in children.

- To examine the effect of comorbid physical and mental health conditions on $\mathrm{CHQ}$. Any effect on QoL in Chinese children aged $\leq 14$ with comorbidly health condition.

\section{Key messages}

- There was significant association of physical and mental health conditions, either individually or comorbidly, on the various concepts of $\mathrm{CHQ}$ in a representative sample of Chinese children aged $\leq 14$ years in Hong Kong.

- Children with mental health problems were apparently more affected than those with physical health problems.

- Chronic renal disease and congenital malformation were the physical conditions associated with the worst QoL in children aged $5-10$ years and children aged 10-14 years, respectively, while behavioural problem was the mental health conditions associated with the worst QoL CHQ in both age groups.

measurement of the impact of having more than one condition on health and well-being are needed to provide surveillance framework for promotion and preventive health programmes of population health. ${ }^{1}$ While there have been substantial studies that focused on a particular physical illness on QoL of individual patients, studies of the impact on QoL due to multiple conditions which occur more commonly in children ${ }^{1}$ or older people ${ }^{2}$ were scarce and furthermore there is no report on Chinese children. We therefore conducted a study to examine the association of different physical and mental 


\section{ARTICLE SUMMARY}

Strengths and limitations of this study

- One of the largest studies using a child self-report QoL measure in a population-based study.

- Use of both child self-report when age warranted and parent reported for younger ages within a study with fairly concurrent result is another substantial strength.

- Parental reports of physician diagnosed health conditions might underestimate the prevalence of various health conditions.

- Possibility of over-rating about certain health concepts like family activities, family cohesion as it was difficult to keep absolute confidentiality in children aged 10-14 years who reported $\mathrm{CHQ}$ themselves due to in general small household size in Hong Kong.

- Our study result may not be directly applicable to Chinese elsewhere as the living environment and standard, societal influence, social value, etc are different.

conditions, individually or comorbidly, on the general health $(\mathrm{GH})$ and well-being of Chinese children in Hong Kong (HK).

\section{METHODS}

\section{Sampling strategy and sample size}

The Child Health Survey (CHS) was a population-based cross-sectional in-person household health survey of Chinese children aged $\leq 14$ years in HK. The survey was carried out from September 2005 to August 2006. The target sample size was set to be approximately 7500 subjects. Details of sampling methodology are delineated in CHS Report 2005-2006, Department of Health, HK. ${ }^{3}$ In brief, sampling units were selected using systematic replicated samples based on the frame of quarters maintained by the Census and Statistics Department at the first stage. At the second stage, households with children aged $\leq 14$ residing were selected for the interview and all children aged $\leq 14$ in the sampled households were included. The survey was approved by the Ethics Committee of Department of Health, HK. Written consent was obtained from parents.

\section{Survey instruments}

We obtained information from a parent as proxy respondent for children aged under 11 , and both directly from the child and from a parent for those aged 11-14. We conducted face-to-face interview with parents of all participants to obtain household, family and personal demographic characteristics. We measured the GH status using the Child Health Questionnaire (CHQ). CHQ was developed in the USA for the measurement of physical and psychosocial well-being of children aged 5 and older. ${ }^{4}$ Parents of children aged 5-10 completed the validated Chinese version of the CHQ-parent form CHQ-PF50 and children aged 11-14 completed the validated Chinese version of the CHQ-child form
CHQ-CF87, respectively, without the help of the interviewer. $^{5}$ The CHQ-PF50 and CHQ-CF87-consist of 50 and 87 items, based on which 14 multidimensional health concepts are measured. These concepts include: $\mathrm{GH}$ perceptions, physical functioning $(\mathrm{PF})$, role functioning-emotional (RE), role functioningbehavioural (RB), role functioning-physical (RP), bodily pain (BP), general behavioural (BE), mental health $(\mathrm{MH})$, self-esteem (SE), family activities (FA), family cohesion (FC), parental impact-emotional (PE), parental impact-time (PT) and change in health $(\mathrm{CH})$. The two concepts, $\mathrm{RE}$ and $\mathrm{RB}$ are combined into one, namely role functioning-emotional and behavioural (REB) in the CHQ-PF50. Two concepts, PE and PT, are not applicable for CHQ-CF87 as the questionnaire is answered by the children themselves. These health concepts comprise multiple items or a single item. With the exception of the GH scale, the global behaviour item and the family cohesion item, the response to these items are based on a 4-week recall period. The response options of each item vary from 4-6 levels. For concepts with multiple items, the responses to the items are summed up and transformed to a scale that ranges from 0 (lowest possible score indicating the worst health) to 100 (highest possible score indicating the best health). Family cohesion, the only health concept comprising a single item in both CHQ-PF50 and CHQ-CF87, is also transformed to a $0-100$ scale. We did not analyse $\mathrm{CH}$ in our study as this health concept was not validated in our previous Chinese version. We included questions on the prevalence of selected important chronic health conditions that had ever been diagnosed by a doctor, which included cardiorespiratory conditions, growth and endocrine conditions, neurological and developmental conditions, blood, oncology, immunology conditions, etc. The choices of these health conditions were based on local expert opinion, available local and overseas data on disease prevalence and CHSs of other countries. ${ }^{6}$

\section{Statistical methods and analysis}

We described the population characteristics with proportions and means and estimated the prevalence of chronic health conditions by age group of 5-10 years and 11-14 years. We used hierarchal linear regression ${ }^{1}$ to explore the associations between chronic health conditions with each concept of the CHQ of the two age groups in two separate analysis. We put the physical and mental conditions as independent variables and as confounder. Each concept of the parent-reported or selfreported $\mathrm{CHQ}$ as dependent variables into the model with adjustment of potential confounders including child's gender and age, parents' martial status, parents' employment status and parents' education level. The size of the unstandardised regression coefficients indicated the mean difference between participants with that particular health condition and those without that health condition. The lower the score for CHQ concept, indicating worse health status as attained by the 
participants with a particular health condition compared with those without it, the more negative value would be the regression coefficients. $p$ Values of $<0.05$ were considered as significant. Analyses were conducted using SAS Software, V.9.1. (Cary, North Carolina, USA).

We used multiple analyses of variance (MANOVA) to evaluate the attributing effect of isolated physical health condition and that of isolated mental health condition on various concepts of the $\mathrm{CHQ}$ when compared with children without any health conditions. We also employed MANOVA to examine the effect of comorbid physical and mental health conditions on various concepts of CHQ. We categorised the participants into three groups: reported either one physical or one mental health condition; reported two comorbid physical and mental health conditions and reported three or more comorbid physical and mental health conditions. We adjusted the potential confounders including child's gender and age, parents' marital status, parents' employment status and parents' educational level. A p value of $<0.01$ was used in the post hoc tests following the MANOVA.

\section{RESULTS}

The demographic characteristics of the sample are shown in table 1 . There were 3223 children (1541 girls and 1682 boys) aged 5-10 years (mean $7.8 \pm 1.7$ years) and 2657 children (1268 girls and 1389 boys) aged 11-14 years (mean $12.5 \pm 1.2$ years). There were slightly more parents had no or primary level of education for the younger age group than the older age group $(7.9 \%$ vs $13.3 \%)$.

Table 2 shows the prevalence of health conditions tabulated by age group and gender. Mild-to-moderate vision problems (including short-sightedness, longsightedness, astigmatism and colour-blindness), allergic rhinitis, eczema, food allergies and asthma were the top five health conditions reported in both age groups. Vision problems were more prevalent in women and in the older age group. Allergic rhinitis, food allergies and asthma were more prevalent in men in either age group. The prevalence of eczema was similar across either sex in children aged 5-10 years but more prevalent in women aged 11-14 years. Attention deficit hyperactivity disorder (ADHD) and then developmental delay were the commonest mental health conditions reported in both age groups with the conditions more prevalent in men than women.

Table 3 categorises the participants by age and by the number of reported health conditions: no physical or mental health condition; reported either one physical or one mental health condition; reported two comorbid physical and mental health conditions and reported

Table 1 Demographic characteristics of children of the two age groups

\begin{tabular}{|c|c|c|c|}
\hline Characteristics & $\begin{array}{l}5-10 \text { years } \\
(n=3223) \\
N(\%)\end{array}$ & $\begin{array}{l}11-14 \text { years } \\
(n=2657) \\
N(\%)\end{array}$ & $\begin{array}{l}\chi^{2} \\
p \text { Value }\end{array}$ \\
\hline Sex & & & 0.996 \\
\hline Female & $1541(47.8)$ & $1268(47.7)$ & \\
\hline Male & $1682(52.2)$ & 1389 (52.3) & \\
\hline Age & & & - \\
\hline Mean (SD) & $7.8(1.7)$ & $12.5(1.1)$ & \\
\hline Parent marital status & & & 0.037 \\
\hline Single & $4(0.1)$ & $2(0.1)$ & \\
\hline Married & 3036 (94.2) & $2456(92.4)$ & \\
\hline Divorced/separated & $121(3.8)$ & $119(4.5)$ & \\
\hline Widow & $46(1.4)$ & $62(2.3)$ & \\
\hline Cohabitant & $8(0.3)$ & $13(0.5)$ & \\
\hline Missing & $8(0.3)$ & $5(0.2)$ & \\
\hline Mother working status & & & 0.183 \\
\hline Yes & $1528(47.4)$ & $1196(45.0)$ & \\
\hline No & $1647(51.1)$ & $1421(53.5)$ & \\
\hline Missing & $48(1.5)$ & $40(1.5)$ & \\
\hline Father working status & & & 0.003 \\
\hline Yes & $2902(90.0)$ & $2303(86.7)$ & \\
\hline No & $167(5.2)$ & $183(6.9)$ & \\
\hline Missing & $154(4.8)$ & $171(6.4)$ & \\
\hline Highest education level of parents & & & $<0.0001$ \\
\hline No and primary & $256(7.9)$ & 354 (13.3) & \\
\hline Secondary and matriculation & $2348(72.9)$ & $1950(73.4)$ & \\
\hline Tertiary & 578 (17.9) & 315 (11.9) & \\
\hline Missing & $41(1.3)$ & $38(1.4)$ & \\
\hline
\end{tabular}


Table 2 Prevalence of specific health conditions by age group and gender

\begin{tabular}{|c|c|c|c|c|}
\hline \multirow[b]{2}{*}{ Health concern } & \multicolumn{2}{|l|}{$5-10$ years } & \multicolumn{2}{|l|}{$11-14$ years } \\
\hline & $\begin{array}{l}\mathrm{n}(\%) \\
\text { Female } \\
(\mathrm{n}=1541)\end{array}$ & $\begin{array}{l}\text { Male } \\
(n=1682)\end{array}$ & $\begin{array}{l}\mathrm{n}(\%) \\
\text { Female } \\
(\mathrm{n}=1268)\end{array}$ & $\begin{array}{l}\text { Male } \\
(n=1389)\end{array}$ \\
\hline \multicolumn{5}{|l|}{ Mental } \\
\hline $\begin{array}{l}\text { Developmental delay or mental retardation + } \\
\text { learning problems }\end{array}$ & $11(0.71$ & $19(1.13)$ & $6(0.47)^{\star}$ & $19(1.37)^{*}$ \\
\hline Attention Deficit Hyperactivity Disorder & $10(0.65)^{*}$ & $25(1.49)^{*}$ & $4(0.32)$ & $12(0.86)$ \\
\hline Behavioral problems & $1(0.06)^{*}$ & $11(0.65)^{*}$ & $4(0.32)$ & $8(0.58)$ \\
\hline Anxiety problems + depression & $3(0.19)$ & $2(0.12)$ & $5(0.39)$ & $4(0.29)$ \\
\hline Autism & $0(0)$ & $3(0.18)$ & $3(0.24)$ & $1(0.07)$ \\
\hline \multicolumn{5}{|l|}{ Physical } \\
\hline $\begin{array}{l}\text { Vision (short, long sight, astigmatism and colour } \\
\text { blindness) }\end{array}$ & $343(22.26)^{\mathrm{a}}$ & $357(21.22)^{a}$ & $576\left(45.43^{a}\right.$ & $602\left(43.34^{a}\right.$ \\
\hline Allergic rhinitis & $323(20.96)^{\star \star \star}$ & $494(29.37)^{\star \star \star}$ & $286(22.56)^{\star \star \star}$ & $428(30.81)^{\star \star \star}$ \\
\hline Eczema + other skin problem & $182(11.81)$ & $201(11.95)$ & $147(11.59)$ & $144(10.37)$ \\
\hline Food allergies & $62(4.02)$ & 89 (5.29) & $57(4.5)$ & $67(4.82)$ \\
\hline Asthma & $57(3.7)$ & $83(4.93)$ & $56(4.42)$ & $67(4.82)$ \\
\hline $\begin{array}{l}\text { Growth problem (too short, too tall, too thin, and too } \\
\text { fat) }\end{array}$ & $30(1.95)$ & $30(1.78)$ & $19(1.5)$ & 29 (2.09) \\
\hline Chronic respiratory, lung or breathing trouble & $20(1.3)^{*}$ & $39(2.32)^{*}$ & $11(0.87)^{\star}$ & $27(1.94)^{*}$ \\
\hline Speech problems & $24(1.56)$ & $38(2.26)$ & $14(1.1)^{\star}$ & $26(1.87)^{*}$ \\
\hline Cerebral palsy or other motor problems & $10(0.65)$ & $11(0.65)$ & $10(0.79)$ & $11(0.79)$ \\
\hline $\begin{array}{l}\text { Vision problems (serious problem, congenital } \\
\text { cataract and amblyopia, blind) }\end{array}$ & $14(0.91)$ & $16(0.95)$ & $16(1.26)$ & $10(0.72)$ \\
\hline Anaemia and other blood diseases & $12(0.78)$ & $10(0.59)$ & $6(0.47)$ & $7(0.5)$ \\
\hline Hearing impairment or deafness & $12(0.78)$ & $12(0.71)$ & $6(0.47)$ & $7(0.5)$ \\
\hline Chronic orthopaedic, bone or joint problems & $1(0.06)$ & $2(0.12)$ & $5(0.39)$ & $4(0.29)$ \\
\hline Gastrointestinal and liver problems & $5(0.32)$ & $5(0.3)$ & $2(0.16)$ & $3(0.22)$ \\
\hline Epilepsy (seizure disorder) & $2(0.13)$ & $2(0.12)$ & $4(0.32)$ & $3(0.22)$ \\
\hline $\begin{array}{l}\text { Sleep disturbance (including obstructive sleep } \\
\text { apnoea) }\end{array}$ & $3(0.19)$ & $2(0.12)$ & $2(0.16)$ & $2(0.14)$ \\
\hline $\begin{array}{l}\text { Cardiac diseases (excluding congenital heart } \\
\text { disease) }\end{array}$ & $5(0.32)$ & $5(0.3)$ & $2(0.16)$ & $1(0.07)$ \\
\hline Endocrine problems (eg, thyroid) & $0(0)$ & $3(0.18)$ & $2(0.16)$ & $1(0.07)$ \\
\hline Diabetes & $0(0)$ & $0(0)$ & $0(0)$ & $1(0.07)$ \\
\hline Chronic renal disease & $0(0)$ & $1(0.06)$ & $2(0.16)$ & $1(0.07)$ \\
\hline Cancer & $1(0.06)$ & $1(0.06)$ & $0(0)$ & $0(0)$ \\
\hline Primary immunodeficiency & $0(0)$ & $0(0)$ & $0(0)$ & $0(0)$ \\
\hline Chronic rheumatic disease (eg, JIA and SLE) & $2(0.13)$ & $1(0.06)$ & $1(0.08)$ & $0(0)$ \\
\hline $\begin{array}{l}\text { Congenital malformation (eg, cleft lip/palate; } \\
\text { including } \mathrm{CHD} \text { ) }\end{array}$ & $1(0.06)$ & $3(0.18)$ & $1(0.08)$ & $0(0)$ \\
\hline
\end{tabular}

three or more comorbid physical and mental health conditions. For children aged 5-10 years, around $70 \%$ of the parents reported that their children did not have any physical or mental health condition, $29 \%$ reported that their children had one physical health condition, $1 \%$ reported that their children had one mental health condition and $1.2 \%$ reported that their children had comorbid physical and mental health conditions. Fifty-two per cent of children aged 11-14 years reported no physical or mental health condition, $46 \%$ reported one physical health condition, $0.3 \%$ reported one mental health condition and $1.4 \%$ reported comorbid physical and mental health condition. Physical health condition was more prevalent than mental health condition in either age group.

Table 4 shows that in children aged 5-10 years, 2 of 5 mental health conditions $(40 \%)$ and 18 of $22(82 \%)$ physical health conditions were found to have negative effect on various concepts of CHQ scores after adjusting for presence of other health conditions and potential confounders. The regression coefficients indicating the mean difference in CHQ scores between participants with a particular health condition and those without that health condition ranged from -1.1 to -53.7 . The top three physical conditions with the most negative regression coefficients were chronic renal disease 
Table 3 Number (\%) of children with physical, mental and both health conditions

\begin{tabular}{lc} 
Number of concerns & $\begin{array}{c}\text { Number of } \\
\text { children (\%) }\end{array}$ \\
\hline Children aged $5-10$ years & $2081(69.2)$ \\
No physical or mental health & $858(28.5)$ \\
condition & $26(0.8)$ \\
One physical & $16(0.5)$ \\
One mental & \\
Two conditions (one physical and & $22(0.7)$ \\
mental) & \\
Three or more conditions & \\
combination of physical and & \\
mental health condition & $1252(52.1)$ \\
Children aged 11-14 years & \\
No physical or mental health & $1106(46.0)$ \\
condition & $8(0.3)$ \\
One physical & $16(0.6)$ \\
One mental & \\
Two conditions (one physical and & \\
mental) & $20(0.8)$ \\
Three or more conditions & \\
combination of physical and & \\
mental health condition & \\
\hline
\end{tabular}

( -53.7 on $\mathrm{PE}$ and -28 on $\mathrm{PF})$; cancer $(-28$ on $\mathrm{RP},-25$ on $\mathrm{RB},-21.5$ on $\mathrm{PF}$ ) and congenital malformations ( -27 on FA and -20.2 on $\mathrm{RB}$ ) while the mental health condition with the most negative regression coefficients were behavioural problems (-25.9 on PE, -24.7 on PT, -22 on FA, -19 on REB, -16.3 on BE, -15.6 on RP and -13.8 on $\mathrm{MH}$ ). With regard to the extent of the effect, asthma, growth problem and speech problems were associated with negative effect on eight concepts of $\mathrm{CHQ}$. Whereas behavioural problems, allergic rhinitis, cerebral palsy or other motor problems on seven and eczema on six of $\mathrm{CHQ}$ concepts.

As shown in table 5, children aged 11-14 years also reported were apparently less affected than younger children, with 3 of 5 mental health conditions $(60 \%)$ and 11 of $22(50 \%)$ physical health conditions having negative effect on various concepts of CHQ scores. The regression coefficients ranged from -1.3 to -40.5 . The top three physical conditions with the most negative regression coefficients were congenital malformation $(-40.5$ on $\mathrm{BP})$; diabetes $(-34.3$ on $\mathrm{GH})$ and cardiac diseases ( -23 on FA and -18.6 on $\mathrm{MH})$. Behavioural problem was also the mental health condition having the most negative regression coefficient. For the extent of effect, growth problem was associated with negative effect on five concepts of $\mathrm{CHQ}$ followed by mild vision problem and behavioural problems on four concepts.

Table 6 showed the different CHQ scores for health concepts in children of the two different age groups. For single health condition, children reported either one physical or one mental condition were compared with children reported neither physical nor mental problem. For comorbid health conditions, children reported one physical and one mental health condition and children reported three or more comorbid physical and mental health conditions were compared with children reported either one physical or one mental condition.

For children aged 5-10 years, MANOVA showed a significant effect of physical and mental health condition on different scores for $\mathrm{CHQ}$ concepts $(\mathrm{F}(24,5746)=3.76$, $\mathrm{p}<0.0001)$. There was an apparent, yet statistically not significant trend that those with isolated mental health problem had the lowest scores across all concepts of CHQ among the three groups (table 7). Post hoc tests using a significance $\mathrm{p}<0.01$ demonstrated that children with isolated mental condition reported significantly lower scores in 2 (REB, BE) of 12 concepts than those with isolated physical condition and those without any reported health condition. Children with isolated physical condition reported significantly lower scores on 2 (BE and PT) of 12 concepts than those without any reported health condition. Significant impact of physical and mental health condition on scores for CHQ concepts was also observed in children aged 11-14 years (F $(22,4058)=3.45, \mathrm{p}<0.0001)$. Similar to the younger age group, there was an apparent but statistically nonsignificant trend that those with isolated mental health condition had the lowest scores across all concepts of CHQ except in RE (table 7). Compared with children without any health condition, children with isolated physical condition reported significantly lower scores on 5 (PF, BP, RE, BE and FA) of 11 concepts and children with isolated mental condition reported significantly lower score in $\mathrm{RE}(\mathrm{p}<0.01)$.

Table 8 showed the MANOVA and post hoc test results for the effect of comorbid physical and mental health conditions on scores of CHQ concepts in the children of the two different age groups. MANOVA showed a significant effect of comorbid physical and mental health conditions on scores of $\mathrm{CHQ}$ concepts in both children aged $5-10$ years $(\mathrm{F}(241786)=2.85 ; \mathrm{p}<0.0001)$ and in children aged $11-14$ years $(\mathrm{F}(221970)=1.67 ; \mathrm{p}<0.02777)$. For the younger age group, post hoc tests demonstrated that children with three more comorbid physical and mental health conditions reported significantly lower scores in 7 of $11 \mathrm{CHQ}$ concepts (GH, PF, BP, REB, FA, $\mathrm{PE}$ and $\mathrm{PT}$ ) than those with one physical or one mental health condition; and one (BP) of $11 \mathrm{CHQ}$ concepts than those with two comorbid physical and mental health condition. For children age 11-14 years, those with three or more comorbid physical and mental health conditions reported significantly lower scores in 1 of $11 \mathrm{CHQ}$ concepts (RE) than those with one physical or one mental health condition.

\section{DISCUSSION}

To the best of our knowledge, this is the first study of the association of physical and mental health conditions, 
Table 4 Mean difference in health-related quality of life concepts scores among children aged 5-10 years with specific mental or physical health conditions

\begin{tabular}{|c|c|c|c|c|c|c|c|c|c|c|c|c|}
\hline Predictors & GH & PF & $\mathbf{R P}$ & BP & REB & BE & MH & SE & FC & PE & PT & $\begin{array}{l}\text { Total } \\
\text { domains }\end{array}$ \\
\hline \multicolumn{13}{|l|}{ Mental } \\
\hline Behavioral problems & & & -15.6 & & -19 & -16.3 & -13.8 & -22 & & -25.9 & -24.7 & 7 \\
\hline Attention deficit hyperactivity disorder & & & & & -9.4 & & & -6.3 & & & & 2 \\
\hline Anxiety problems + Depression & & & & & & & & & & & & 0 \\
\hline Autism & & & & & & & & & & & & 0 \\
\hline $\begin{array}{l}\text { Developmental delay or mental retardation + Learning } \\
\text { problems }\end{array}$ & & & & & & & & & & & & 0 \\
\hline \multicolumn{13}{|l|}{ Physical } \\
\hline Chronic renal disease & & -28 & & & & & & & & -53.7 & & 2 \\
\hline Cancer & & -21.5 & -28 & & -25 & & & & & & & 3 \\
\hline Congenital malformation (eg, cleft lip/palate; including CHD) & & & & & -20.2 & & & -27 & & & & 2 \\
\hline Epilepsy (seizure disorder) & & & & & & & & & -18.5 & & -21.2 & 2 \\
\hline Sleep disturbance (including obstructive sleep apnoea) & & & & & -23 & & & -12.7 & -20.2 & & & 3 \\
\hline Endocrine problems (eg, thyroid) & & & -17.9 & & -19 & & & & & & & 2 \\
\hline Cardiac diseases (excluding congenital heart disease) & & & -9.4 & & & & & & & & & 1 \\
\hline Cerebral palsy or other motor problems & -6.6 & -6.3 & -9.4 & -10.2 & -15.5 & & -7.3 & -7.9 & & & & 7 \\
\hline Anaemia and other blood diseases & & & & & & -6.3 & & & & & & 1 \\
\hline Hearing impairment or deafness & & & & & & & -6.3 & & & & & 1 \\
\hline Growth problem (too short, too tall, too thin and too fat) & -5 & -4.2 & -5 & & -4.8 & & -4.9 & & -6.8 & -6.9 & -9.8 & 8 \\
\hline Chronic respiratory, lung; or breathing trouble & & & & -3.9 & & -3.9 & & & & -6.3 & -7.5 & 4 \\
\hline Asthma & -7.9 & & & & & -4.5 & -3.7 & $-3.7-3.9$ & -5.6 & -6.6 & -6.3 & 8 \\
\hline Speech problems & -6.4 & & -6.9 & & -8.7 & -4.6 & & $-4.8-8.3$ & & -12.3 & -12.8 & 8 \\
\hline Eczema + other skin problem & & & & -3.5 & -2.4 & -2.4 & & -3.4 & & -3.7 & -3.4 & 6 \\
\hline Food allergies & -2.7 & & & & & & & & & & & 1 \\
\hline Allergic rhinitis & & & -1.3 & -1.4 & -1.4 & -1.2 & & -1.5 & & -2.2 & -2.1 & 7 \\
\hline Vision (short, long sight, astigmatism and colour blindness) & & & & & & -1.9 & & $-1.1-1.3$ & & & & 3 \\
\hline Chronic rheumatic disease (eg, JIA, SLE) & & & & & & & & & & & & 0 \\
\hline Gastrointestinal and liver problems & & & & & & & & & & & & 0 \\
\hline Chronic orthopaedic, bone, or joint problems & & & & & & & & & & & & 0 \\
\hline Vision problems (Serious problem) & & & & & & & & & & & & 0 \\
\hline Diabetes & & & & & & & & & & & & 0 \\
\hline
\end{tabular}

All figures displayed are statistically significant at the 0.05 level.

BE, general behaviour; BP, bodily pain; CHD; congenital heart defects; FA, family activities; FC, family cohesion; GH, general health; JIA, juvenile idiopathic arthritis; SLE, systemic lupus erythematosus; MH, mental health; PE, parental impact-emotional; PF, physical functioning; PT, parental impact-time; REB, role functioning-emotional and behavioural; RP, role erythematosus; $\mathrm{MH}$, mental health; $\mathrm{P}$ 
Table 5 Mean difference in health-related quality of life concepts scores among children aged 11-14 years with specific mental or physical health conditions

\begin{tabular}{|c|c|c|c|c|c|c|c|c|c|c|c|}
\hline Predictors & GH & PF & RP & BP & RE & RB & BE & MH & SE FA & FC & $\begin{array}{l}\text { Total } \\
\text { domains }\end{array}$ \\
\hline \multicolumn{12}{|l|}{ Mental } \\
\hline Behavioral problems & -11.0 & & & & & & & -13.0 & -23.7 & -19.5 & 4 \\
\hline Attention deficit hyperactivity disorder & & & & & -16.2 & & -14.3 & & & & 2 \\
\hline Anxiety problems + depression & & & & & & & & & & & 0 \\
\hline Autism & & & & & & & & & & & 0 \\
\hline Developmental delay or mental retardation + Learning problems & -8.9 & -14.2 & -10.0 & & & & & & & & 3 \\
\hline \multicolumn{12}{|l|}{ Physical } \\
\hline Chronic renal disease & & & & & & & & & & & 0 \\
\hline Cancer & & & & & & & & & & & 0 \\
\hline Congenital malformation (eg, cleft lip/palate; including CHD) & & & & -40.5 & & & & & & & 1 \\
\hline Epilepsy (seizure disorder) & & & & & & & & & & & 0 \\
\hline Sleep disturbance (including obstructive sleep apnoea) & & & & & & & & & & & 0 \\
\hline Endocrine problems (eg, thyroid) & & & & & & & & & & & 0 \\
\hline Cardiac diseases (excluding congenital heart disease) & & & & & & & & -18.6 & -23 & & 2 \\
\hline Cerebral palsy or other motor problems & & & & & & & & & & & 0 \\
\hline Anaemia and other blood diseases & & & & & & & & & & & 0 \\
\hline Hearing impairment or deafness & & & & & & -14.1 & -8.0 & & & & 2 \\
\hline Growth problem (too short, too tall, too thin and too fat) & -7.8 & -6.1 & & -10.2 & & & & -7.0 & & -6.9 & 5 \\
\hline Chronic respiratory, lung; or breathing trouble & & & & & & & & & & & 0 \\
\hline Asthma & -4.9 & & & & & & & & -3.6 & & 2 \\
\hline Speech problems & & -9.7 & & & & & -5.5 & & -9.2 & & 3 \\
\hline Eczema + other skin problem & & & & & & & -1.4 & & -2.4 & & 2 \\
\hline Food allergies & & & & & & & & & & & 0 \\
\hline Allergic rhinitis & & -2.2 & & -4.1 & -3.2 & -1.9 & -2.4 & & -2.4 & & 6 \\
\hline Vision (short, long sight, astigmatism and colour blindness) & & -1.6 & & -2.0 & & & -1.3 & & -1.3 & & 4 \\
\hline Chronic rheumatic disease (eg, JIA and SLE) & & & & & & & & & & & 0 \\
\hline Gastrointestinal and liver problems & & & -18.0 & & -21.2 & -21.3 & & & & & 3 \\
\hline Chronic orthopaedic, bone or joint problems & & & & & & & & & & & 0 \\
\hline Vision problems (serious problem) & & & & & & & & & & & 0 \\
\hline Diabetes & -34.3 & & & & & & & & & & 1 \\
\hline
\end{tabular}

All figures displayed are statistically significant at the 0.05 level.

$\mathrm{BE}$, general behaviour; BP, bodily pain; CHD, congenital heart defects; FA, family activities; FC, family cohesion; GH, general health; JIA, juvenile idiopathic arthritis; SLE, systemic lupus erythematosus; MH, mental health; PE, parental impact-emotional; PF, physical functioning; PT, parental impact-time; REB, role functioning-emotional and behavioural; RP, role functioning-physical; SE, self-esteem. 
Table 6 Child Health Questionnaire (CHQ) scores across physical or mental and comorbid health conditions

\begin{tabular}{|c|c|c|c|c|c|c|}
\hline & \multicolumn{3}{|c|}{ Single health condition } & \multicolumn{3}{|c|}{ Comorbid health conditions } \\
\hline & $\begin{array}{l}{ }^{\star} \text { No physical } \\
\text { or mental }\end{array}$ & $\begin{array}{l}\text { One } \\
\text { physical }\end{array}$ & $\begin{array}{l}\text { One } \\
\text { mental }\end{array}$ & $\begin{array}{l}{ }^{\star} \text { One physical } \\
\text { or mental }\end{array}$ & $\begin{array}{l}\text { One physical } \\
\text { and one mental }\end{array}$ & $\begin{array}{l}\text { Three combine } \\
\text { conditions }\end{array}$ \\
\hline \multicolumn{7}{|l|}{ Age $5-10$ years } \\
\hline General health & 70.0 & 69.8 & 64.8 & 69.6 & 68.1 & 57.1 \\
\hline Physical functioning & 94.8 & 94.4 & 88.9 & 94.2 & 90.0 & 84.9 \\
\hline $\begin{array}{l}\text { Role functioning- } \\
\text { physical }\end{array}$ & 95.5 & 95.0 & 89.1 & 94.9 & 88.9 & 90.5 \\
\hline Bodily pain & 96.6 & 95.5 & 93.1 & 95.5 & 97.3 & 84.8 \\
\hline $\begin{array}{l}\text { Role functioning- } \\
\text { emotional and } \\
\text { behavioural }\end{array}$ & 94.3 & 93.5 & 77.8 & 93.1 & 84.4 & 76.2 \\
\hline General behaviour & 83.4 & 81.1 & 72.8 & 80.9 & 79.2 & 73.7 \\
\hline Mental health & 85.7 & 85.0 & 82.5 & 85.0 & 89.3 & 77.9 \\
\hline Self-esteem & 69.7 & 68.4 & 66.0 & 68.3 & 69.3 & 62.1 \\
\hline Family activities & 93.4 & 91.8 & 88.0 & 91.7 & 88.1 & 79.0 \\
\hline Family cohesion & 75.6 & 74.9 & 68.3 & 74.7 & 70.3 & 69.5 \\
\hline $\begin{array}{l}\text { Parental impact- } \\
\text { emotional }\end{array}$ & 80.3 & 78.5 & 70.8 & 78.3 & 75.0 & 57.5 \\
\hline Parental impact-time & 87.5 & 84.5 & 76.9 & 84.3 & 77.0 & 61.4 \\
\hline \multicolumn{7}{|l|}{ Age $11-14$ years } \\
\hline General health & 69.4 & 68.9 & 64.8 & 68.8 & 69.4 & 63.5 \\
\hline Physical functioning & 89.6 & 87.4 & 88.4 & 87.4 & 74.8 & 76.8 \\
\hline $\begin{array}{l}\text { Role functioning- } \\
\text { physical }\end{array}$ & 93.9 & 91.9 & 76.2 & 91.8 & 90.0 & 76.8 \\
\hline Bodily pain & 91.6 & 88.7 & 82.9 & 88.7 & 96.0 & 90.0 \\
\hline $\begin{array}{l}\text { Role functioning- } \\
\text { emotional }\end{array}$ & 90.3 & 87.6 & 66.7 & 87.4 & 83.3 & 67.7 \\
\hline $\begin{array}{l}\text { Role functioning- } \\
\text { behavioural }\end{array}$ & 92.9 & 90.6 & 81.0 & 90.5 & 90.0 & 76.8 \\
\hline Behaviour & 90.1 & 88.4 & 79.3 & 88.3 & 85.9 & 84.2 \\
\hline Mental health & 79.7 & 79.4 & 91.3 & 79.5 & 76.1 & 70.2 \\
\hline Self-esteem & 66.6 & 67.0 & 69.9 & 67.0 & 60.0 & 68.3 \\
\hline Family activities & 92.4 & 90.2 & 91.1 & 90.2 & 93.8 & 81.1 \\
\hline Family cohesion & 68.8 & 67.4 & 62.9 & 67.4 & 71.0 & 68.6 \\
\hline
\end{tabular}

either individually or comorbidly, on the GH and wellbeing of Chinese children in a representative population sample. We show that the QoL of the participants and their parents are affected in multiple concepts in the presence of significant health problems. Children with an isolated mental health problem are apparently more affected than those with an isolated physical health problem. Having more health problems concurrently is associated with worse QoL. As CHS included a fairly representative sample of the target population ${ }^{3}$; thus the result could be generalised to children aged 5-14 in HK.

Mild-to-moderate vision problems, allergic rhinitis, eczema, food allergies and asthma were the top five health conditions reported in both age groups. Our results supported a local study which showed that the prevalence and progression of myopia in HK schoolaged children was much higher than children in Western countries. ${ }^{7}$ While there is evidence supporting genetic influence, the rapid increase in prevalence of myopia in Chinese children over past few decades suggested an important interplay of environmental factors. ${ }^{8}{ }^{9}$ Yet studies that tried to elucidate these environmental factors, such as accommodative demand associated with near-work tasks such as schoolwork, yielded mixed results. ${ }^{10}$ More research in this area is warranted for disease prevention. We found that allergic rhinitis was highly prevalent in children in $\mathrm{HK}$, being the second commonest chronic health condition. Previous ISAAC study showed that prevalence of allergic rhinitis in children aged 6-7 years in HK ranked the second highest among the participating cities or countries. ${ }^{11}$ The high prevalence of allergic rhinitis was postulated to be related to outdoor environmental pollutants in our locality. ${ }^{12}$

ADHD was the most commonly reported mental health problem in both age groups. While our results concurred with recent studies from other countries such as Australia and the USA, ${ }^{13}{ }^{14}$ which also showed that ADHD was the most prevalent mental health problems based on parent-report, our prevalence was much lower 
Table 7 Effect of single physical health condition or mental health condition on Child Health Questionnaire (CHQ) scores for children age 5-10 and 11-14 years

\begin{tabular}{|c|c|c|c|}
\hline \multicolumn{4}{|l|}{ Post hoc tests $(p<0.01)$} \\
\hline Age $5-10$ years (CHQ PF50) & & & $F(24,5746)=3.76 ; p<0.0001$ \\
\hline Role functioning-emotional and behavioural & 0 vs 2 & 1 vs 2 & \\
\hline General behaviour & 0 vs 1,2 & 1 vs 2 & \\
\hline Parental impact-time & 0 vs 1 & & \\
\hline Age $11-14$ years (CHQ CF87) & & & $F(22,4058)=3.45 ; p<0.0001$ \\
\hline Physical functioning & 0 vs 1 & & \\
\hline Bodily pain & 0 vs 1 & & \\
\hline Role functioning-emotional & 0 vs 1,2 & & \\
\hline Bodily pain & 0 vs 1 & & \\
\hline Family activities & 0 vs 1 & & \\
\hline
\end{tabular}

0 , No physical or mental health condition; 1 , one physical; 2 , one mental.

Results were adjusted for sex, age, parents' marital status, parents' employment status and parents' educational level.

(ranged from 4.8\% in Germany between 2003 and 2006 to $9.5 \%$ in the USA in 2007). ${ }^{15}$ Whether the observed difference is due to cultural acceptance or genetic predisposition awaits further evaluation.

Our study showed that our child population has been enjoying fairly good health with low reported prevalence of many physical and mental diseases, which was not unexpected as in many developed countries or cities. Nowadays, the concepts of highest attainable standard of health is not limited to improved survival, free of disease, the ability to perform daily activities but to wellbeing and QoL. In children and adolescents, HRQoL is a multidimensional construct covering physical, emotional, mental, social and behavioural components of well-being as perceived by the children and adolescents or their parents. The definition of HRQoL used for adults thus cannot be directly applied to them. Although there were concern whether children and adolescents could reliably express their own opinions, attitudes and feelings about HRQoL in the past, QoL questionnaires in children and adolescents have been increasingly developed, validated and shown that children and adolescents are able to report on their well-being and functioning. ${ }^{16}$ In fact, our study might be one of the largest studies using a child self-report QoL measure in a population based study and it supported the adoption of child self-report QoL questionnaire in addressing the issue of HRQoL in children and adolescents in the future. The use of both child self-report when age warranted and parent reported for younger ages within a study with fairly concurrent result is also a substantial strength of our study. There have been several studies showing the impact of individual health conditions on child health and well-being compared with healthy children using either a generic or specific type of QoL questionnaire. ${ }^{17-22}$ A generic instrument allows comparison across children with different health conditions and healthy population samples while a disease-specific type can enhance sensitivity for concepts relevant to a particular health condition. However, studies comparing QoL across different health conditions were difficult to conduct. Most studies that adopted generic questionnaires such as CHQ or PedQL would thus examine patients of a specific health condition with healthy population samples in a single study and then compare other health conditions using the

Table 8 Effect of comorbid physical health conditions and mental health conditions on Child Health Questionnaire (CHQ) scores for children age $5-10$ and $11-14$ years

\begin{tabular}{lc}
\hline Post hoc tests $(\boldsymbol{p}<\mathbf{0 . 0 1})$ & \\
\hline Age 5-10 years (CHQ PF50) & 0 vs 2 \\
General health & 0 vs 2 \\
Physical functioning & 0 vs 2 \\
Bodily pain & 0 vs 2 \\
Role functioning-emotional and behavioural 0 vs 2 \\
Family activities & 0 vs 2 \\
Parental impact-emotional & 0 vs 2 \\
Parental impact-time & \\
Age 11-14 years (CHQ CF87) & 0 vs 2 \\
Role functioning-emotional &
\end{tabular}

$F(24,1786)=2.85 ; p<0.0001$

0 vs 2

vs $2 \quad 1$ vs 2

0, One physical or one mental health condition; 1 , one physical and one mental; 2 , three combine conditions.

Results were adjusted for sex, age, parents' marital status, parents' employment status and parents' educational level. 
existing database. Limitations included retrospective nature of comparison groups and failure to adjust or match the demographic characteristics across the groups. Our large population study partially reduced these limitations although caution needs to be exercised when interpreting the result of health conditions of very low prevalence. Our study showed that children with either mental or physical health conditions reported lower scores across more than one concept of $\mathrm{CHQ}$ that measure physical or psychosocial functioning. Chronic renal diseases, cancer and congenital malformation had the most significant negative effect on health-related QoL in children aged 5-10 years. The respective health conditions in children aged 11-14 years were congenital malformation, diabetes and cardiac diseases. Our study was similar to the findings of a recent cross-sectional, multicentre cohort study. It showed that children aged 2-18 years with renal dialysis had the lowest scores of all three concepts of PedQL when comparing with those with liver transplant, renal transplant, juvenile rheumatoid arthritis, type 1 diabetes, cancer in remission and cardiac disease. ${ }^{23}$

We found that children aged $5-10$ years with an isolated mental health problem appeared to be more affected in their QoL when compared with either normal or children with an isolated physical health problem. In particular, the scores in concepts that measured general behaviour and role functioning in emotions and behaviour were significantly lower. Our result is consistent with previous studies in Western population. ${ }^{24}$ Of interest, parents of children with isolated physical health conditions reported statistically significant lower scores in concept that measured parental impact on time but not for those parents with isolated mental health conditions. Such a trend was not observed in older children who reported CHQ scores by themselves.

Older children with an isolated physical health condition seemed to be more affected than those with an isolated mental health condition. The former had lower scores in concepts that measured physical health aspects, mental health aspects and family impact related to activities while the latter had lower score in concepts that measure role functioning-emotional only when compared with those without any. Unexpectedly, there were apparent higher scores in concepts that measured mental health, self-esteem and family impact on activities in those with isolated mental condition. We believe that the observation could be due to the small number of children with an isolated mental health problem that might have skewed the result rather than being a genuine effect.

Our study also supported other studies $^{7}{ }^{11}$ which reported that having a greater number of health problems is associated with poorer QoL across different $\mathrm{CHQ}$ concepts. The effect was more prominent in our children aged 5-10 years. Those with three or more comorbid health conditions were significantly more affected than those with two health conditions in one concept and those with only one health condition in seven concepts. In older children aged 11-14 years, those with three comorbid health conditions were significantly more affected than those with one health condition in one concept only.

There were limitations to our study which require attention regarding drawing conclusions: first, we used parental reports of physician diagnosed health conditions to estimate the prevalence. The findings may be different from the reported prevalence by physicians; however, parental reports have been widely adopted in population health study. Moreover, previous studies showed that self-report measures of health conditions are fairly accurate. ${ }^{25}$ Our results also concurred favourably with previous local condition-specific prevalence studies. ${ }^{7}{ }^{11}$ We did not attempt to analyse individual health conditions. For some very low prevalent health conditions such as diabetes, rheumatic diseases, the results are based on a small sample size. Second, we could not ensure absolute confidentiality in children aged 10-14 years who reported CHQ themselves due to in general small household size in our local population. The possibility of over-rating certain health concepts such as family activities, family cohesion could not be completely ruled out. Lastly, while our study suggests that there may be possible cultural differences in disease acceptance and perception of QoL between our HK Chinese and Caucasians, it may not be directly applicable to Chinese elsewhere as the living environment and standards, societal influence, social values, etc are different.

\section{CONCLUSION}

Our study shows important information concerning the prevalence of different health conditions and its association on the QoL in a representative sample of Chinese children in HK. The information may be used for comparing the impact of various conditions and for measurement of the impact of having more than one condition on health and well-being so as to provide a surveillance framework for promotion and preventive health programmes.

Acknowledgements The authors thank the Department of Health, Hong Kong for commissioning of the Child Health Survey; all the participants and their parents; Ms. Rita So for her secretarial support. They are grateful to The Doris Zimmern Charitable Foundation for supporting their Community Child Health Programme.

Contributors SLL and LY contributed to conception and design of the paper. All authors contributed to interpretation of data. WHSW contributed analysis of the data. SLL wrote the original paper draft. All authors contributed equally to the revising and approval of the final version of the paper.

Funding This research received no specific grant from any funding agency in the public, commercial or not-for-profit sectors.

Competing interests None.

Patient consent Obtaied

Ethics approval Institutional Review Board of the University of Hong Kong.

Provenance and peer review Not commissioned; externally peer reviewed. 
Data sharing There are no additional data available.

\section{REFERENCES}

1. Waters E, Davis E, Nicolas C, et al. The impact of childhood conditions and concurrent morbidities on child health and well-being. Child Care Health Dev 2008;34:418-29.

2. Chen $\mathrm{H}$, Cohen $\mathrm{P}, \mathrm{Kasen} \mathrm{S}$, et al. Impact of adolescent mental disorders and physical illnesses on quality of life 17 years later. Arch Pediatr Adolesc Med 2006;160:93-9.

3. Department of Health, HKSAR (Internet). Hong Kong: Child Health Survey 2005/2006. http://www.chp.gov.hk/files/pdf/chs_0506_eng. pdf (accessed 23 Nov 2012)

4. Landgraf JM, Abetz L, Ware JE. (1999, 1996). The CHQ: a user's manual (2nd printing). Boston, MA: HealthAct. 1st printing. Boston, MA: The Health Institute. (No longer in print. Now available as Child health Questionnaire Scoring and Interpretation Manual. 2008 HealthActCHQ Inc., Cambridge, MA, USA.

5. $\mathrm{Ng}$ JY, Landgraf JM, Chiu CS, et al. Preliminary evidence on the measurement properties of the Chinese version of the Child Health Questionnaire, parent form (CHQ-pF50) and child form (CHQ-CF87). Qual Life Res 2005;14:1775-81.

6. New South Wales Child Health Survey 2001. N S W Public Health Bull 2002;13(Suppl 3):1-84.

7. Fan DS, Lam DS, Lam RF, et al. Prevalence, incidence, and progression of myopia of school children in Hong Kong. Invest Ophthalmol Vis Sci 2004;45:1071-5.

8. Wu MM, Edwards $\mathrm{MH}$. The effect of having myopic parents: an analysis of myopia in three generations. Optom Vis Sci 1999;76:387-92.

9. Edwards MH, Lam CS. The epidemiology of myopia in Hong Kong. Ann Acad Med Singapore 2004:33:34-8.

10. Lu B, Congdon N, Liu X, et al. Associations between near work, outdoor activity, and myopia among adolescent students in rural China: the Xichang Pediatric Refractive Error Study report no. 2. Arch Ophthalmol 2009;127:769-75.

11. Asher Ml, Montefort S, Bjorksten B, et al. Worldwide time trends in the prevalence of symptoms of asthma, allergic rhinoconjunctivitis, and eczema in childhood: ISAAC Phases One and Three repeat multicountry cross-sectional surveys. Lancet 2006;368:733-43.

12. Lee SL, Wong W, Lau YL. Increasing prevalence of allergic rhinitis but not asthma among children in Hong Kong from 1995 to 2001 (Phase 3 International Study of Asthma and Allergies in Childhood). Pediatr Allergy Immunol 2004;15:72-8.
13. Increasing prevalence of parent-reported attention-deficit/ hyperactivity disorder among children-United States, 2003 and 2007. MMWR Morb Mortal Wkly Rep 2010;59:1439-43.

14. Efron $D$, Hazell $P$, Anderson V. Update on attention deficit hyperactivity disorder. J Paediatr Child Health 2011;47:682-9.

15. Huss $\mathrm{M}$, Holling $\mathrm{H}$, Kurth $\mathrm{BM}$, et al. How often are German children and adolescents diagnosed with ADHD? Prevalence based on the judgment of health care professionals: results of the German health and examination survey (KiGGS). Eur Child Adolesc Psychiatry 2008;17(Suppl 1):52-8.

16. Solans M, Pane S, Estrada MD, et al. Health-related quality of life measurement in children and adolescents: a systematic review of generic and disease-specific instruments. Value Health 2008;11:742-64.

17. McKenna AM, Keating LE, Vigneux A, et al. Quality of life in children with chronic kidney disease-patient and caregiver assessments. Nephrol Dial Transplant 2006;21:1899-905.

18. Engelen V, Koopman HM, Detmar SB, et al. Health-related quality of life after completion of successful treatment for childhood cancer Pediatr Blood Cancer 2011;56:646-53.

19. Pike NA, Evangelista LS, Doering LV, et al. Clinical profile of the adolescent/adult Fontan survivor. Congenit Heart Dis 2011;6:9-17.

20. Ronen GM, Streiner DL, Rosenbaum P. Health-related quality of life in children with epilepsy: development and validation of self-report and parent proxy measures. Epilepsia 2003;44:598-612.

21. Yam WK, Ronen GM, Cherk SW, et al. Health-related quality of life of children with epilepsy in Hong Kong: how does it compare with that of youth with epilepsy in Canada? Epilepsy Behav 2008;12:419-26.

22. Juniper EF, Guyatt $\mathrm{GH}$, Feeny $\mathrm{DH}$, et al. Measuring quality of life in children with asthma. Qual Life Res 1996;5:35-46.

23. Limbers CA, Neighbors K, Martz K, et al. Health-related quality of life in pediatric liver transplant recipients compared with other chronic disease groups. Pediatr Transplant 2011;15:245-53.

24. Sawyer MG, Whaites L, Rey JM, et al. Health-related quality of life of children and adolescents with mental disorders. J Am Acad Child Adolesc Psychiatry 2002;41:530-7.

25. Kriegsman DM, Penninx BW, van Eijk JT, et al. Self-reports and general practitioner information on the presence of chronic diseases in community dwelling elderly. A study on the accuracy of patients' self-reports and on determinants of inaccuracy. J Clin Epidemiol 1996;49:1407-17 